\title{
Substrate-induced changes in microbial community-level physiological profiles and their application to discriminate soil microbial communities
}

\author{
CHEN Jian $^{1}$, XIE Huijun ${ }^{1}$, ZHUANG Xuliang ${ }^{1,2, *}$, ZHUANG Guoqiang ${ }^{1}$, \\ BAI Zhihui ${ }^{1}$, ZHANG Hongxun ${ }^{1}$ \\ 1. Research Center for Eco-Environmental Sciences, Chinese Academy of Sciences, Beijing 100085, China. \\ E-mail: chenjian305@mails.gucas.ac.cn \\ 2. Bureau of Science and Technology for Resources and Environment, Chinese Academy of Sciences, Beijing 100864, China
}

Received by 20 August 2007; revised 17 September 2007; accepted 20 September 2007

\begin{abstract}
The addition of simple substrates could affect the microbial respiration in soils. This substrate-induced respiration is widely used to estimate the soil microbial biomass, but little attention has been paid to its influence on the changes of community-level physiological profiles. In this study, the process of microbial communities responding to the added substrate using sole-carbon-source utilization (BIOLOG) was investigated. BIOLOG is biased toward fast-growing bacteria; this advantage was taken to detect the prompt response of the active microbial communities to the added substrate. Four soil samples from agricultural fields adjacent to heavy metal mines were amended with $L$-arginine, citric acid, or $D$-glucose. Substrate amendments could, generally, not only increase the metabolic activity of the microbial communities, but also change the metabolic diverse patterns compared with no-substrate control. By tracking the process, it was found that the variance between substrate-induced treatment and control fluctuated greatly during the incubation course, and the influences of these three substrates were different. In addition, the application of these induced changes to discriminate soil microbial communities was tested. The distance among all samples was greatly increased, which further showed the functional variance among microbial communities in soils. This can be very useful in the discrimination of microbial communities even with high similarity.
\end{abstract}

Key words: substrate-induced respiration (SIR); BIOLOG; community-level physiological profiles (CLPPs)

\section{Introduction}

The basal respiration (BR) of microbial communities in soils under natural conditions is very low, but the addition of simple substrate, such as glucose, can rapidly increase the microbial respiration and maintain for a period of time. This substrate-induced respiration (SIR) is considered to be proportional to the population of the substrateresponsive biomass and is widely used for estimating the initial microbial biomass in soil (Anderson and Domsch, 1978; Martens, 1995; Mondini et al., 2006). Recently, further kinetic analysis of SIR has shown the dynamics of this process and discriminated two different kinds of responses. They are the normal growth $(r)$ and slow-growing $(K)$ microorganisms according to their different respiration rate (Stenström et al., 1998, 2001). It is also possible to obtain a catabolic diversity by addition of different substrates because of the diverse responses of the community to different substrates (Campbell et al., 2003; Degens and Harris, 1997). Although SIR has been used to estimate the microbial biomass and the kinetics of the carbon dioxide evolved from the system, little attention has been paid

\footnotetext{
*Corresponding author. E-mail: xlzhuang@cashq.ac.cn.
}

to the changes of community-level physiological profiles (CLPPs) induced by the substrate. Degens (1998) proved that addition of simple organic substrates can influence microbial functional diversity by using fingerprints made up of variant amounts of carbon dioxide $\left(\mathrm{CO}_{2}\right)$ induced by a series of selected substrates. Compbell et al. (2003) reported that measurement of SIR with a set of ecologically relevant substrates can abstract more variance than by using BIOLOG GN (Gram-negative) plate.

Sole-carbon-source utilization (BIOLOG) is a commercial system from Biolog Inc., USA initially for the identification of heterotrophic bacterial isolates. This was done by inoculating microorganisms onto a 96-well microplate containing 95 individual carbon sources and a water control. It distinguishes microbial communities based on tetrazolium violet, a kind of redox dye that can be reduced by a diverse of aerobic microbes (Bochner and Savageau, 1977). Garland and Mills (1991) first introduced BIOLOG GN plate to assess the heterotrophic microbial functional diversity. Since then, hundreds of research on characterizing microbial communities were conducted with this method using various kinds of plates (PrestonMafham et al., 2002). BIOLOG ECO (ecology) plate, 
with 31 unique environmentally applicable carbon sources and 3 replicates on each plate, was specially formed for community analysis (Preston-Mafham et al., 2002). The effects of ECO plate to distinguish among microbial communities were proved to be better than other kinds, such as GN2 (Gram-negative) and GP2 (Gram-positive) plate (Classen et al., 2003). There are, however, several problems in the measurement and interpretation of the results. But, it provides a rapid and reproducible means to reflect the function and functional potential within the heterotrophic bacterial community (Campbell et al., 1997; Classen et al., 2003; Garland, 1997; Preston-Mafham et al., 2002). The color development of the BIOLOG system is biased towards fast-growing culturable bacteria, which was widely considered as its limitation (Heuer and Smalla, 1997; Konopka et al., 1998). Nevertheless, the advantage of this characteristic could be taken to detect the prompt responses of the active microbial communities to the added substrate. Besides, investigation of the short time process of substrate-induced response can help better understand community-level metabolism, which is not well studied.

The authors believe that substrate amendment can not only quickly stimulate the respiration of microorganisms measured as $\mathrm{CO}_{2}$, but can also alter their metabolic diversity. This stimulation might fluctuate during a period of time because of the diverse reactions of different bacteria. The classification and characterization of microbial communities are generally based on their variances. Thus, shifts of the metabolic potential might be essential for the discrimination of the microbial communities. In this study, BIOLOG ECO plate was used to trail the process of microbial communities responding to the added simple substrate. It was also used to characterize the power of the substrate-induced effect in discriminating soil microbial communities. According to the authors, this was the first time to trail the consecutive metabolic changes of microbial communities in response to substrate amendment for a period of time.

\section{Materials and methods}

\subsection{Sampling and characteristics of soils}

The upper $20 \mathrm{~cm}$ of four contrasting soils were collected in September, 2006. Soil samples DX1 and DX2 were collected from the agricultural fields adjacent to a copper mine in Dexing City, Jiangxi Province, China $\left(28^{\circ} 57^{\prime} 36^{\prime \prime} \mathrm{N}\right.$, $117^{\circ} 34^{\prime} 48^{\prime \prime}$ E). Soil sample DX1 was collected from a vegetable field just next to the mine, whereas DX2 was collected from rice field about $10 \mathrm{~km}$ away from the mine. Samples NJT1 and NJT2 were collected from rice fields near a lead-zinc mine in Duyun City, Guizhou Province, China $\left(26^{\circ} 43^{\prime} 12^{\prime \prime} \mathrm{N}, 107^{\circ} 31^{\prime} 48^{\prime \prime} \mathrm{E}\right)$. The relative geographic position of NJT1 and NJT2 was similar with DX1 and DX2 samples. All samples were placed in plastic bags and transported to laboratory as soon as possible. After passing through a 2-mm sieve, thoroughly mixed, samples were stored at $4^{\circ} \mathrm{C}$ until further use.

The main physical and chemical characteristics of soils are shown in Table 1. Water content, $\mathrm{pH}$, organic carbon, total nitrogen, available potassium, and readily available phosphorus were measured using previous methods (Chen et al., 2007). The total concentration of copper $(\mathrm{Cu})$, lead $(\mathrm{Pb})$, and zinc $(\mathrm{Zn})$ was also measured by an inductively coupled plasma-optical emission spectrometer (OPTIMA 2000, Perkin Elmer Co., USA) (Tessier et al., 1979). Just like their comparative distances to the heavy metal mines, sample DX1 showed an extremely high level of $\mathrm{Cu}$ concentration and sample NJT1 showed a high level of $\mathrm{Pb}$ and $\mathrm{Zn}$ concentration (Table 1).

\subsection{Substrate-induced respiration}

Referring to former work (Campbell et al., 2003), Larginine, citric acid, and $D$-glucose were selected as the inducing substrates. These substrates represented three different kinds of important carbon sources: amino acid, carboxylic acid, and carbohydrate. Ten grams (dry weight) of each soil was weighed into a $15-\mathrm{ml}$ vial. Four milliliters of $L$-arginine, citric acid, or $D$-glucose solution with a concentration of $14.5,16$, or $15 \mathrm{mg} / \mathrm{ml}$, respectively, was added to maintain equal amount of organic carbon addition. No-substrate control was set with the same mixing and moisture addition. Then, the vial was placed into a respirometric jar containing $50 \mathrm{ml}$ of $0.04 \mathrm{~mol} / \mathrm{L} \mathrm{NaOH}$ to trap the $\mathrm{CO}_{2}$ evolved. Sealed with screw caps, jars were incubated at $28^{\circ} \mathrm{C}$ for $2 \mathrm{~h}$. After incubation, $\mathrm{NaOH}$ was titrated by $\mathrm{HCl}$ with an automatic potential titrator (716 DMS Titrino, Metrohm Co., Switzerland) to fix both BR and SIR. All treatments were in three replicates.

\subsection{BIOLOG analysis}

The CLPP induced by $L$-arginine, citric acid, $D$-glucose or without amendment were determined in BIOLOG ECO microplates (Biolog Inc., Hayward, USA). Ten grams (dry weight) soil amended with simple substrate or without induction was added to $100 \mathrm{ml}$ sterile $0.85 \%$ saline solution. The samples were shaken on a micro shaker at full speed (2000 r/min) for $1 \mathrm{~min}$ and then were placed on ice for $1 \mathrm{~min}$. This was repeated 3 times and then settled for 5 min. Five milliliters of each suspension was diluted 1,000

Table 1 Main physical and chemical characteristics of soils used

\begin{tabular}{lllllrl}
\hline $\begin{array}{l}\text { Soil } \\
\text { sample }\end{array}$ & $\mathrm{pH}$ & $\begin{array}{l}\text { Water } \\
(\%, W / W)\end{array}$ & $\begin{array}{l}\text { Organic carbon } \\
(\%, W / W)\end{array}$ & $\begin{array}{l}\text { Total nitrogen } \\
(\%, W / W)\end{array}$ & $\begin{array}{l}\mathrm{Cu} \\
(\mathrm{mg} / \mathrm{kg})\end{array}$ & $\begin{array}{l}\mathrm{Pb} \\
(\mathrm{mg} / \mathrm{kg})\end{array}$ \\
\hline DX1 & 5.5 & 22 & 8.43 & 0.21 & $1,194.3$ & 23.6 \\
DX2 & 4.6 & 34 & 5.45 & 0.21 & 61.8 & 116.6 \\
NJT1 & 6.8 & 28 & 6.28 & 0.25 & 46.9 \\
NJT2 & 6.7 & 24 & 6.54 & 0.24 & 53.6 & 307.5 \\
\hline
\end{tabular}


times in a 10-fold dilution series, from which $150 \mu \mathrm{l}$ of supernatant was inoculated to each well. The plates were incubated in the dark at $28^{\circ} \mathrm{C}$ and color development was measured at a wavelength of $590 \mathrm{~nm}$ every $24 \mathrm{~h}$ for $7 \mathrm{~d}$ using an automated microtiter-plate reader (Biolog Inc., Hayward, USA).

\subsection{Statistical analysis}

Induced respirations of all the microbial communities were compared within each substrate ( $L$-arginine, citric acid, and $D$-glucose) by one way ANOVA (analysis of variance). An $\alpha$ level of 0.05 was used for the statistical test. The means of average well color development (AWCD) (Garland and Mills, 1991) for the three replicates on the same plate were calculated and then compared among the different treatments in each soil. The AWCD can give an overall trend of metabolic activity of the microbial communities over the time measured. To compare the CLPPs, principal component analysis (PCA) was first performed based on the variance-covariance matrix. The first 10 principal components were used for the following canonical variate analysis (CVA). PCA reduce the dimensionality of the BIOLOG data to a small enough scale for CVA. The mean Mahalanobis distance for all groups was used to measure the overall separation effect of the groups. All statistical analyses were performed by using GenStat 8.1 (VSN International Ltd., Oxford, UK).

\section{Results}

\subsection{Changes of soil respiration induced by different substrate}

The results of SIR are shown in Fig.1. Although all substrate amendments raised respiration rate, one-way ANOVA indicated that not all of the inductions were significant different compared with no-substrate control. The effects of $L$-arginine or $D$-glucose on different soils were generally the same. As for citric acid, there were great variances among the four soil samples. The performance was equivalent in soil samples DX1 and DX2, NJT2 was higher than DX1 and DX2, whereas NJT1 was the highest.

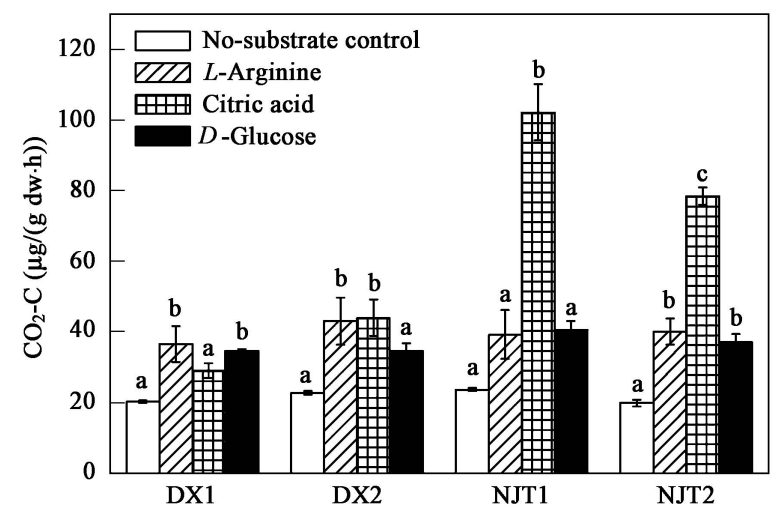

Fig. 1 Respiration of microbial communities induced by $L$-arginine, citric acid and $D$-glucose compared with no-substrate control in four soil samples. The error bars indicate standard error. Different characters in the same group indicate significant difference (LSD, $\alpha=0.05$ ).

\subsection{Substrate-induced CLPPs}

The overall AWCD of no-substrate control exhibited great variance (Fig.2), which might correlate with the level of heavy metals. Sample DX2 was the least contaminated, thus was the highest in microbial activity. The activity of DX1 was only a little lower than DX2, although its Cu level was extremely high. The very low metabolic activities of NJT1 and NJT2 were likely caused by the high level of Pb and $\mathrm{Zn}$ (Table 1).

The influences of the three substrates on the activity of the microbial communities in all the soil samples were also quite different (Fig.2). $L$-Arginine could only increase the metabolic activity of NJT2; citric acid stimulated all of the metabolic activity except in DX2; $D$-glucose eventually obtained higher activity than control in every sample, though not always during the process. Besides, the $D$-glucose induced curves were all generally double sigmoidal (Fig.2), which was not observed in control and other treatments. To determine whether biphasic curves meant that some substrates were used rapidly and others later, the curves of every single substrate in $D$-glucose induced samples (data not shown) were then checked. Apart from the expected greatly protracted curves, sigmoidal ones (Fig.3) were also obtained. These two kinds of distinct curves were not found in control.

We used CVA to evaluate the substrate-induced changes in microbial functional diversity. This multivariate analysis was very useful in reflecting the shifts in CLPPs patterns among different samples. The variance between substrate amended sample and control, to represent the real shifts induced by substrate instead of direct comparison among substrate amended samples, was used. This was because the metabolic diverse pattern might change at different times during BIOLOG measurement (Garland, 1997). Generally, the first 5 principal components could totally explain the variance between substrate amended sample and control (data not shown). In the following CVA, only one canonical variate (CV) was generated. Thus, the mean Mahalanobis distance to signify the variance was directly used. Different soil samples showed variant responses even induced by the same substrate (Fig.4). Some varied sharply during the $168 \mathrm{~h}$ incubation, whereas others did not change greatly. With citric acid amendment, the communities changed greatly at an early stage. The effects of $L$-arginine or $D$-glucose were protracted compared with citric acid. $D$ Glucose even exhibited a second stimulation in DX1 and NJT2.

\subsection{Substrate-induced discrimination of microbial communities}

The discriminating abilities after $L$-arginine, citric acid, and $D$-glucose amendment with no-substrate control among the four soils (Fig.5) were compared. Data sets of both substrate-induced soil sample and its corresponding no-substrate control were acquired at the time when they showed the biggest variance during BIOLOG incubation (Fig.4, Table 2). Shifts in data sets generally did not influence the discrimination of the microbial communities 

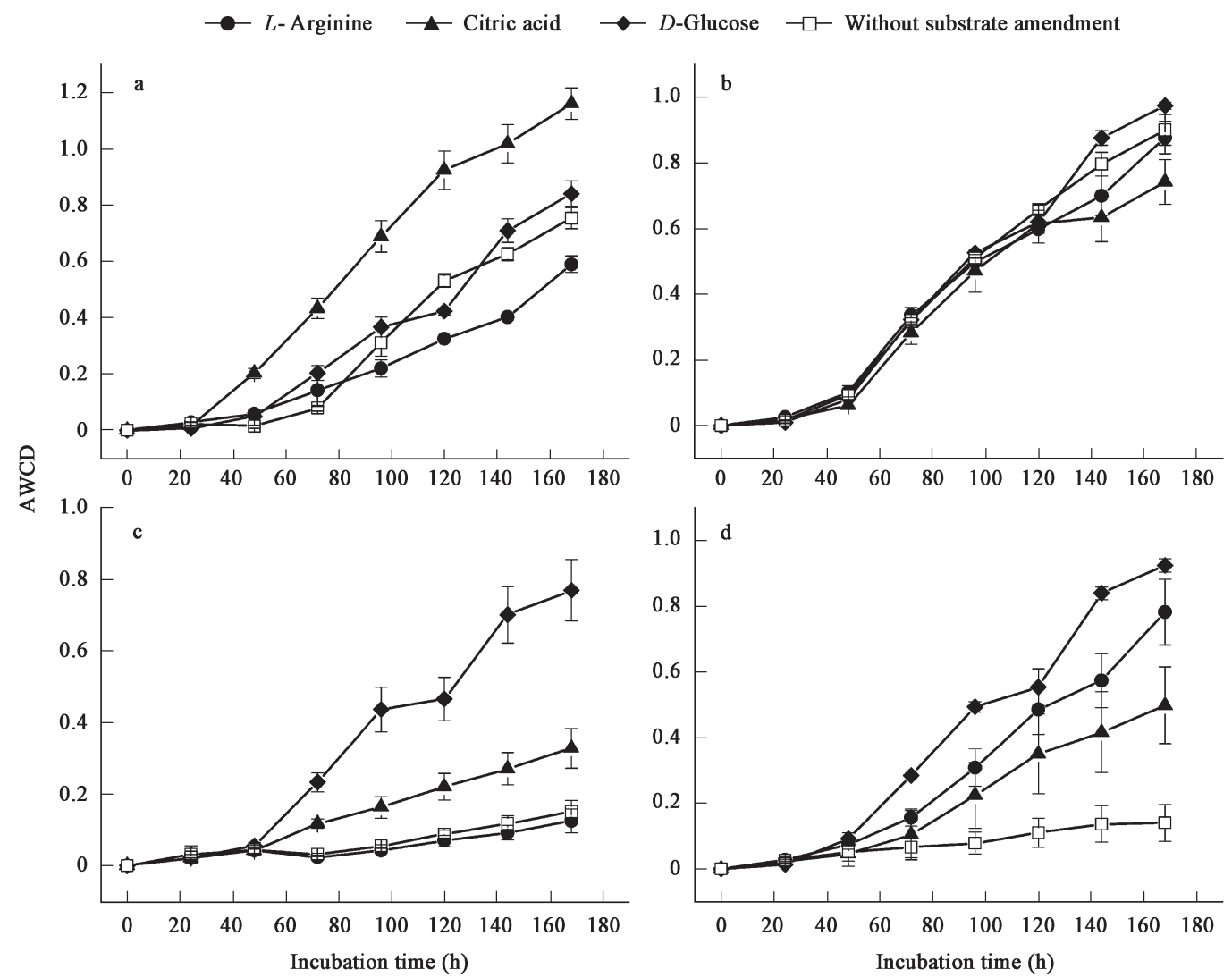

Fig. 2 Average well color development (AWCD) of soil samples DX1 (a), DX2 (b), NJT1 (c), and NJT2 (d). The error bars indicate standard error.
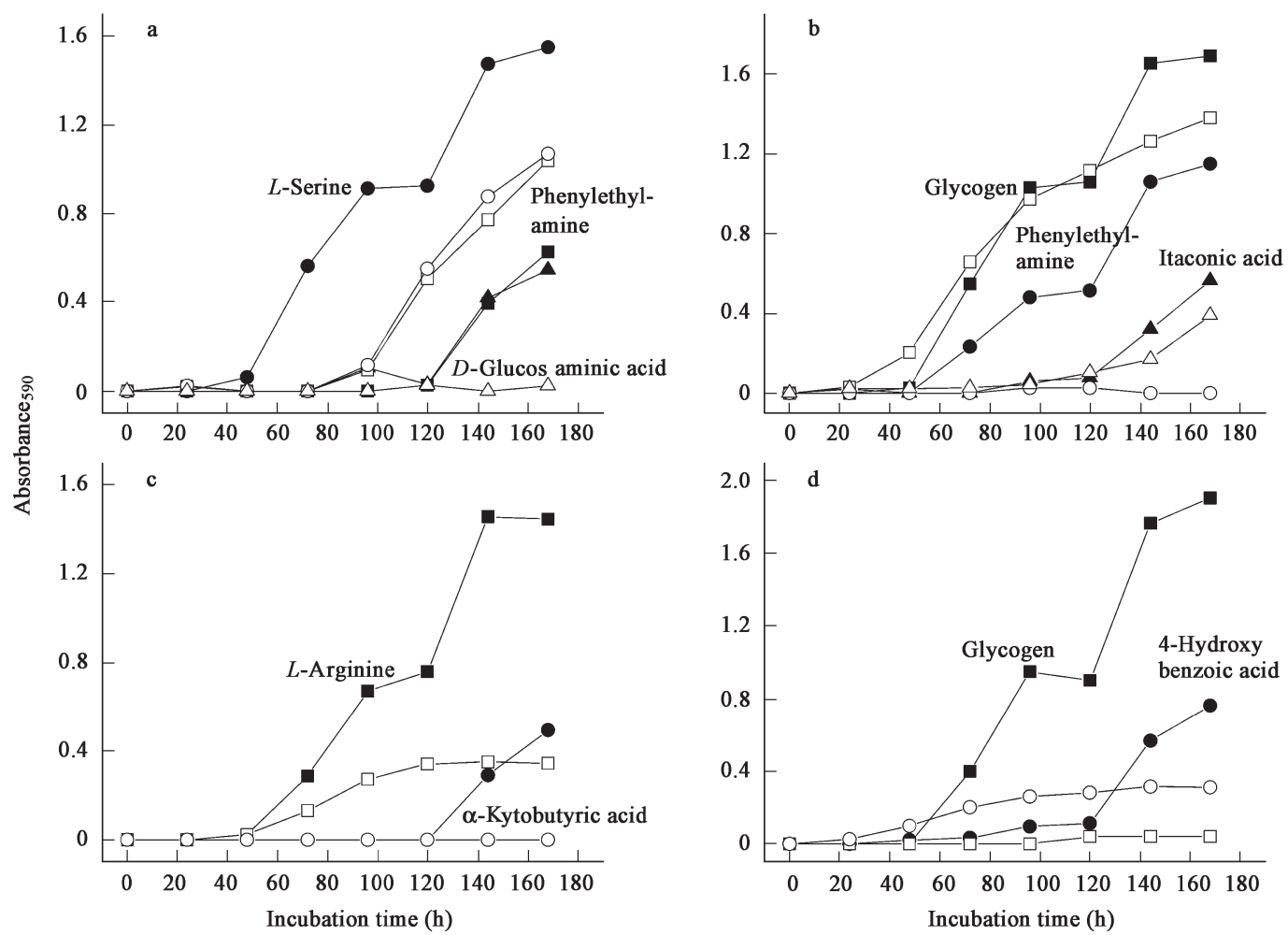

Fig. 3 Comparison of the color development of several substrates on BIOLOG plate between $D$-glucose induced samples (gray symbols) and no-substrate control (open symbols) in soil samples DX1 (a), DX2 (b), NJT1 (c), and NJT2 (d). 

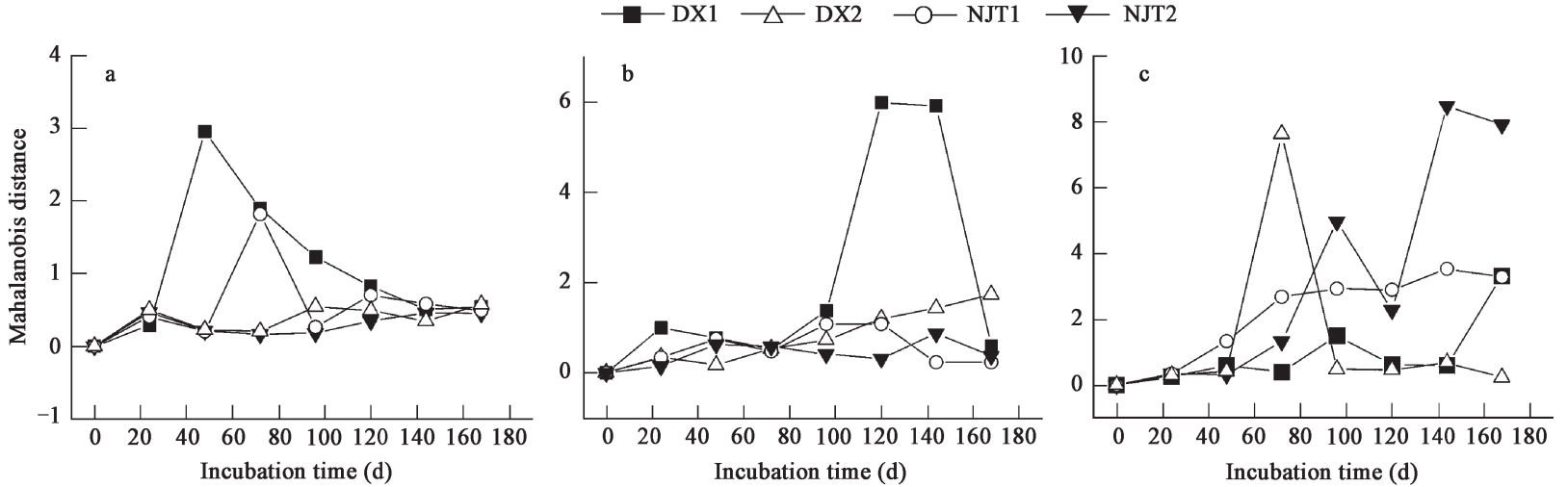

Fig. 4 Variation of mean Mahalanobis distance between samples induced by citric acid (a), $L$-arginine (b), D-glucose (c) with no-substrate control, respectovely, in four soil samples at different times during BIOLOG incubation.
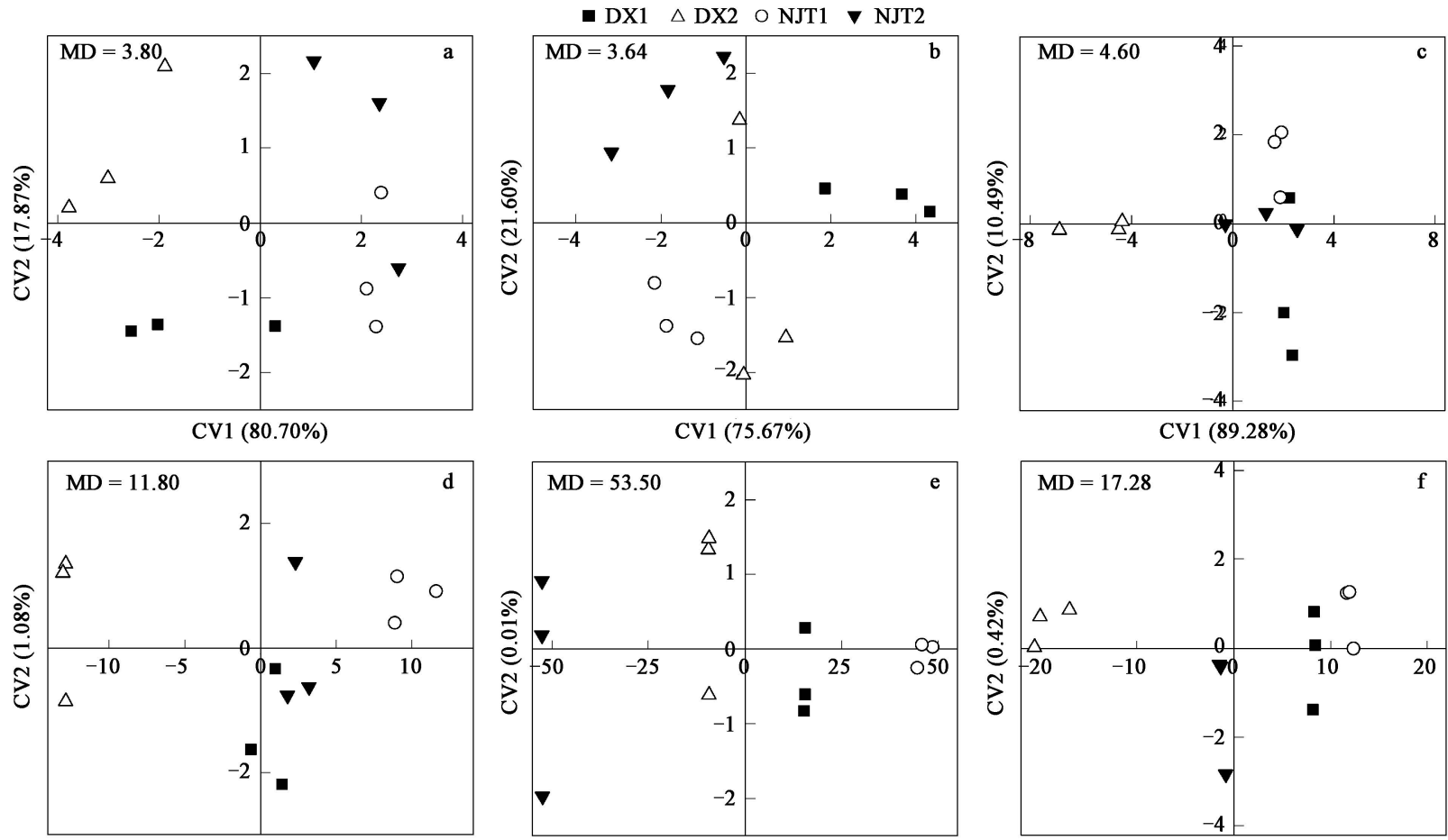

CV1 (98.88\%)

CV1 $(99.97 \%)$

CV1 (99.55\%)

Fig. 5 Plot of first and second CVs induced by $L$-arginine (d), citric acid (e), $D$-glucose (f), and their corresponding controls (a, b, c) for four soil samples. MD: Mahalanobis distance among all groups.

Table 2 Time during BIOLOG incubation used for the comparison of discriminating ability between substrate-induced samples and corresponding no-substrate control

\begin{tabular}{llll}
\hline Soil sample & L-Arginine (h) & Citric acid (h) & $D$-Glucose (h) \\
\hline DX1 & 120 & 48 & 168 \\
DX2 & 168 & 96 & 72 \\
NJT1 & 120 & 72 & 168 \\
NJT2 & 144 & 120 & 144 \\
\hline
\end{tabular}

in all controls by CVA (Figs.5a, 5b, and 5c). In contrast, the mean Mahalanobis distances were significantly increased to $11.80,53.50$, and 17.28 when amended with $L$-arginine, citric acid, and D-glucose (Figs.5d, 5e, and 5f), which indicated higher discriminating abilities after being induced by these substrates.

\section{Discussion}

\subsection{Substrate-induced changes in CLPPs}

The four samples were all heavily contaminated with various heavy metals (Table 1). But, only sample NJT1 and NJT2 with high $\mathrm{Pb}$ concentration showed very low metabolic activity and the activity of DX1 was very high even with extremely high $\mathrm{Cu}$ concentration (Fig.2). It is possible that the microbes in DX1 are tolerant with high $\mathrm{Cu}$ concentration or that most of the $\mathrm{Cu}$ element exists in inactive phase, which has little effect on the microbial metabolism. The long time induced effects of $L$-arginine, citric acid, and $D$-glucose were investigated on both microbial activity and functional diversity level with BIOLOG microplate. Although the short time induction $(2 \mathrm{~h})$ was not obvious in some samples (Fig.1), both metabolic activity 
and diversity showed variances with substrate amendment in contrast to no-substrate control afterwards (Figs.2 and 4). The consecutive automated record of the data helped to monitor the metabolic process of the microbial communities on various environmentally related substrates. The effects of the added substrates can be divided into two groups. Soil samples DX1 and DX2, with a comparative high metabolic activity (Figs.2a and 2b), generally showed no big variance with substrate amendment. In contrast, the metabolic activity of soil samples NJT1 and NJT2 greatly increased except for $L$-arginine amendment in NJT1 (Figs.2c and 2d). This contrast indicated that substrate amendment can generally increase the activity of the previously inactive communities. Also, it is possible that the finite amount of each substrate used in ECO plate and the small space in the well may limit our ability to detect the substrate-induced activity of DX1 and DX2.

The substrate-induced CLPPs (Fig.4) were compared with SIR results (Fig.1). It was found that although the respiration of samples with $L$-arginine or $D$-glucose amendment was low in contrast to citric acid, the metabolic potential of the communities may also be immensely changed, especially with $D$-glucose. This was probably because although the induced production of $\mathrm{CO}_{2}$ with $L$-arginine or $D$-glucose was lower than that with citric acid, the metabolic potential of the communities had already been influenced. The complicated influences of simple substrate on microbial communities were partly shown by tracking the process of metabolism on BIOLOG ECO plate. The addition of simple substrate may alter the metabolic diverse pattern without greatly affecting metabolic activity. In all the four soil samples, treatments with substrate amendment can be distinguished from nosubstrate control according to the metabolic patterns. In addition, shifts within the same soil microbial communities induced by different substrates showed great variance, which indicated the distinct responses of the communities to different substrates (Fig.4). It was reported that the physical and chemical properties of the soils appeared to influence SIR (Degens and Harris, 1997). Thus, to exactly evaluate substrate-induced effect under such complicated circumstances remains a problem.

The discovery of double sigmoidal AWCD curve by BIOLOG system in assessing CLPPs was also original, which reflected the complex effect of $D$-glucose on microbial communities. Further investigation showed that the curves of certain substrates were either changed into double sigmoidal, or greatly protracted (Fig.3), which showed that the induction may be more complex than was thought. In addition, the curves of diversity variance between $D$ glucose induced treatment and control had two peaks in soil samples DX1 and NJT2 (Fig.4c). Considering that only part of the substrates on the plate are double sigmoidal or protracted, it is possible that the complicated influence of $D$-glucose on the metabolism of certain substrates are masked by other substrates in soil DX2 and NJT1. BIOLOG being a culture-based method, the stimulated bacteria at early stage during incubation might facilitate the growth of the others later.

\subsection{Using substrate-induced changes to discriminate} soil microbial communities

Changes in microbial communities have profound impacts on ecosystem (Bossio and Scow, 1995). Because of various problems such as small size and morphological similarity of microbes, the classification and characterization of microbial communities, as used in animal and plant, is very difficult (Garland and Mills, 1991). The fast and commercially-available detecting system of BIOLOG can reflect real differences in heterotrophic microbial communities (Haack et al., 1995). This biased reflection toward the fast growing dominant bacteria may mask the variance existing in other parts. Here, it was found that CLPPs of microbial communities can be changed by the induction of simple substrate. Thus, whether this characteristic could be applicable in finding the unrevealed variance was examined. The discriminating ability of BIOLOG system with ECO plate was extremely increased with $L$-arginine, citric acid, and $D$-glucose amendment. The authors believe that the increased discriminating ability came from stimulation of simple substrate. The tracking of the substrate-induced process helped to find the time when the greatest variance was stimulated, which can be very useful in the discrimination of heterotrophic microbial communities with high similarity. Substrate amendment further showed the great functional variance among microbial communities in soils, which was not well understood before. However, how to globally describe this variance and the theoretical basis of this stimulated discrimination remains to be determined.

\section{Conclusions}

The process of the substrate-induced effect on both metabolic activity and diversity level was investigated. The results showed that substrate amendment not only increased the metabolic activity but also changed the diversity patterns. The process of this substrate-induced effect is very complicated and varies greatly with different substrate amendments. These induced changes helped to further understand the functional variance among microbial communities in soils and could be very useful in discriminating microbial communities even with high similarity.

\section{Acknowledgements}

This work was supported by the National Natural Science Foundation of China (No. 30470289, 30670039).

\section{References}

Anderson J P E, Domsch K H, 1978. A physiological method for the quantitative measurement of microbial biomass in soils. Soil Biol Biochem, 10: 215-221.

Bochner B R, Savageau M A, 1977. Generalized indicator plate for genetic, metabolic, and taxonomic studies with microorganisms. Appl Environ Microbiol, 33: 434-444.

Bossio D A, Scow K M, 1995. Impact of carbon and flooding on the metabolic diversity of microbial communities in soils. Appl Environ Microbiol, 61: 4043-4050. 
Campbell C D, Chapman S J, Cameron C M, Davidson M S, Potts J M, 2003. A rapid microtiter plate method to measure carbon dioxide evolved from carbon substrate amendments so as to determine the physiological profiles of soil microbial communities by using whole soil. Appl Environ Microbiol, 69: 3593-3599.

Campbell C D, Grayston S J, Hirst D J, 1997. Use of rhizosphere carbon sources in sole carbon source tests to discriminate soil microbial communities. J Microbiol Methods, 30: 3341.

Chen J, Zhuang X L, Xie H J, Bai Z H, Qi H Y, Zhang H X, 2007. Associated impact of inorganic fertilizers and pesticides on microbial communities in soils. World J Microb Biot, 23: 23-29.

Classen A T, Boyle S I, Haskins K E, Overby S T, Hart S C, 2003. Community-level physiological profiles of bacteria and fungi: plate type and incubation temperature influences on contrasting soils. FEMS Microbiol Ecol, 44: 319-328.

Degens B P, 1998. Microbial functional diversity can be influenced by the addition of simple organic substrates to soil. Soil Biol Biochem, 30: 1981-1988.

Degens B P, Harris J A, 1997. Development of a physiological approach to measuring the catabolic diversity of soil microbial communities. Soil Biol Biochem, 29: 1309-1320.

Garland J L, 1997. Analysis and interpretation of communitylevel physiological profiles in microbial ecology. FEMS Microbiol Ecol, 24: 289-300.

Garland J L, Mills A L, 1991. Classification and characterization of heterotrophic microbial communities on the basis of patterns of community-level sole-carbon-source utilization. Appl Environ Microbiol, 57: 2351-2359.
Haack S K, Garchow H, Klug M J, Forney L J, 1995. Analysis of factors affecting the accuracy, reproducibility, and interpretation of microbial community carbon source utilization patterns. Appl Environ Microbiol, 61: 1458-1468.

Heuer H, Smalla K, 1997. Evaluation of community-level catabolic profiling using BIOLOG GN microplates to study microbial community changes in potato phyllosphere. $J$ Microbiol Methods, 32: 49-61.

Konopka A, Oliver L, Turco R F, 1998. The use of carbon substrate utilization patterns in environmental and ecological microbiology. Microb Ecol, 35: 103-115.

Martens R, 1995. Current methods for measuring microbial biomass C in soil: potentials and limitations. Biol Fert Soils, 19: 87-99.

Mondini C, Cayuela M L, Sanchez-Monedero M A, Roig A, Brookes P C, 2006. Soil microbial biomass activation by trace amounts of readily available substrate. Biol Fert Soils, 42: 542-549.

Preston-Mafham J, Boddy L, Randerson P F, 2002. Analysis of microbial community functional diversity using sole-carbon-source utilisation profiles-a critique. FEMS Microbiol Ecol, 42: 1-14.

Stenström J, Stenberg B, Johansson M, 1998. Kinetics of substrate-induced respiration (SIR): theory. Ambio, 27: 3539.

Stenström J, Svensson K, Johansson M, 2001. Reversible transition between active and dormant microbial states in soil. FEMS Microbiol Ecol, 36: 93-104.

Tessier A, Campbell P G C, Bisson M, 1979. Sequential extraction procedure for the speciation of particulate trace metals. Anal Chem, 51: 844-851. 